\title{
Stability of Schwarzschild black holes in fourth-order gravity revisited
}

\begin{abstract}
Yun Soo Myung*
Institute of Basic Sciences and Department of Computer Simulation, Inje University Gimhae 621-749, Korea

Abstract

We revisit the classical stability of Schwarzschild black hole in fourth-order theories of gravity. On the contrary of the stability of this black hole, it turns out that the linearized perturbations exhibit unstable modes featuring the Gregory-Laflamme instability of five-dimensional black string. This shows clearly an instability of black hole in fourth-order gravity with $\alpha=-\beta / 3$.
\end{abstract}

PACS numbers: $04.70 . \mathrm{Bw}, 04.50 . \mathrm{Kd}$

Typeset Using $\mathrm{AT}_{\mathrm{E}} \mathrm{X}$

*e-mail address: ysmyung@inje.ac.kr 


\section{Introduction}

All black hole solutions found in Einstein gravity must pass the stability test. A black hole solution should be stable against the external perturbations because it stands as a physically realistic object [1]. One decouples the linearized equations and then, manages to arrive at the Schrödinger-type equations for physical fields with their potentials. For nonrotating black holes, the stable or unstable nature of black hole perturbation is determined by the shape of the potential. If all potentials are positive for whole range outside the event horizon, the black hole under the consideration is stable.

However, for black holes found in massive gravity theories, the method is not so straightforward to arrive at the stability of black holes because they have more physical degrees of freedom than two of a massless graviton. Whitt [2] has found that provided both massive spin-0 and spin-2 are non-tachyonic, the Schwarzschild black hole is classically stable in fourth-order gravity. This implies that initial perturbations which are regular at infinity and on the future event horizon cannot grow unboundedly with time.

Very recently, Babichev and Fabbri [3] have shown that massive linearized equation around the Schwarzschild black hole in massive gravity and bimetric theories gives rise to an instability of $l=0$ mode by comparing it with the linearized equation around the black string in five dimensions. It turned out that the bimetric black hole solution is unstable provided $m^{\prime}=m \sqrt{1+1 / \kappa}$ satisfies a bound of $0<m^{\prime}<\frac{\mathcal{O}(1)}{2 M_{\mathrm{S}}}$. In addition, the authors [4] have confirmed this result by considering a more generic framework and extending $l=0$ mode to generic modes.

We wish to point out that the above two have the same linearized equation when replacing the Ricci tensor perturbation $\delta \tilde{R}_{\mu \nu}$ by the metric perturbation $h_{\mu \nu}^{(-)}$. However, the above two give controversy to each other. This is why we will revisit the issue of stability of Schwarzschild black hole in fourth-order theories of gravity. We find that the black hole in fourth-order gravity with $\alpha=-\beta / 3$ is unstable since $m_{2}=1 / \sqrt{\beta}$ may satisfy a bound of $0<m_{2}<\frac{\mathcal{O}(1)}{2 M_{\mathrm{S}}}$. 


\section{Perturbation of black holes in fourth-order gravity}

We start with the fourth-order gravity theory given by [2]

$$
S=\frac{1}{16 \pi} \int d^{4} x \sqrt{-g}\left[R-\alpha R^{2}-\beta R_{\mu \nu} R^{\mu \nu}\right]
$$

with two parameters $\alpha$ and $\beta$. This theory is renormalizable in Minkowski spacetimes [5] and it describes 8 degrees of freedom (DOF) [a massless spin-2 graviton with 2 DOF, a massive spin-2 graviton with 5 DOF, and a massive spin-0 with 1 DOF] [6]. However, the massive graviton suffers from having ghosts. We note that $f(R)$-gravity with $\beta=0$ has 3 DOF (a massless spin-2 graviton and a massive spin-0) without ghost [7].

The Einstein equation takes the form

$$
\begin{aligned}
R_{\mu \nu}-2 \alpha R R_{\mu \nu} & -2 \beta R^{\rho \sigma} R_{\rho \mu \sigma \nu}-\beta \nabla^{2} R_{\mu \nu}+(2 \alpha+\beta) \nabla_{\nu} \nabla_{\mu} R \\
& =\frac{g_{\mu \nu}}{2}\left[R-\alpha R^{2}-\beta R_{\rho \sigma} R^{\rho \sigma}+(4 \alpha+\beta) \nabla^{2} R\right] .
\end{aligned}
$$

It is well-known that Eq.(2.2) provides the Schwarzschild black hole solution

$$
d s_{\mathrm{S}}^{2}=\bar{g}_{\mu \nu} d x^{\mu} d x^{\nu}=-e^{\nu(r)} d t^{2}+e^{-\nu(r)} d r^{2}+r^{2} d \Omega_{2}^{2}
$$

with the metric function

$$
e^{\nu(r)}=1-\frac{2 M_{\mathrm{S}}}{r}
$$

In this case, the trace of (2.2) becomes an equation for the Ricci scalar

$$
2(3 \alpha+\beta) \nabla^{2} R+R=0 .
$$

In the case of $\alpha=-\beta / 3$, there is no scalar graviton $(R=0)[8$.

In order to perform the stability analysis, we usually introduce the metric perturbation around the black hole

$$
g_{\mu \nu}=\bar{g}_{\mu \nu}+h_{\mu \nu}
$$

Hereafter we denote the background quantities with the "overbar". However, in fourthorder gravity, the metric perturbation (2.6) leads to the fourth-order differential equation [9] which is almost impossible to be solved. Instead, we consider the resulting perturbation in Ricci tensor, $\delta R_{\mu \nu}(h)$ whose representation is given by

$$
\delta R_{\mu \nu}(h)=\frac{1}{2}\left(\bar{\nabla}^{\rho} \bar{\nabla}_{\mu} h_{\nu \rho}+\bar{\nabla}^{\rho} \bar{\nabla}_{\nu} h_{\mu \rho}-\bar{\nabla}^{2} h_{\mu \nu}-\bar{\nabla}_{\mu} \bar{\nabla}_{\nu} h\right) .
$$


The linearized equation for Ricci tensor takes the second-order differential form

$$
\begin{aligned}
{\left[\left\{\beta \bar{g}_{\mu \rho} \bar{g}_{\nu \sigma}\right.\right.} & \left.+\left(2 \alpha+\frac{\beta}{2}\right) \bar{g}_{\rho \sigma} \bar{g}_{\mu \nu}\right\} \bar{\nabla}^{2}-\bar{g}_{\mu \nu}(2 \alpha+\beta) \bar{\nabla}_{\rho} \bar{\nabla}_{\sigma} \\
& \left.+\left(2 \beta \bar{R}_{\mu \rho \nu \sigma}-\bar{g}_{\mu \rho} \bar{g}_{\nu \sigma}+\frac{1}{2} \bar{g}_{\rho \sigma} \bar{g}_{\mu \nu}\right)\right] \delta R^{\mu \nu}=0 .
\end{aligned}
$$

Taking the trace of (2.8) leads to the linearized Ricci scalar equation

$$
\left(\bar{\nabla}^{2}-m_{0}^{2}\right) \delta R=0
$$

where the spin-0 mass $m_{0}^{2}$ is given by

$$
m_{0}^{2}=-\frac{1}{2(3 \alpha+\beta)} .
$$

Eq.(2.9) is consistent with that obtained from varying (2.5) directly. Here we may choose $2(3 \alpha+\beta)<0$ and $\beta>0$ for $m_{0}^{2}>0$ and $m_{2}^{2}=1 / \beta>0$. In the case of $\alpha=-\beta / 3$, this scalar graviton is decoupled from the theory, implying that $\delta R=0$.

To simplify the stability analysis, we decompose $\delta R_{\mu \nu}$ into the trace and trace-free parts as

$$
\delta R_{\mu \nu}=\frac{\delta R}{4} \bar{g}_{\mu \nu}+\delta \tilde{R}_{\mu \nu}
$$

where

$$
\bar{g}^{\mu \nu} \delta \tilde{R}_{\mu \nu}=0 .
$$

Then, the linearized equation of (2.8) becomes

$$
\begin{aligned}
& {\left[\bar{g}_{\mu \rho} \bar{g}_{\nu \sigma}\left(\bar{\nabla}^{2}-\frac{1}{\beta}\right)+2 \bar{R}_{\mu \rho \nu \sigma}\right] \delta \tilde{R}^{\mu \nu}} \\
& +\left[\left(\frac{2 \alpha}{\beta}+\frac{3}{4}\right) \bar{g}_{\rho \sigma} \bar{\nabla}^{2}-\left(\frac{2 \alpha}{\beta}+1\right) \bar{\nabla}_{\rho} \bar{\nabla}_{\sigma}+\frac{1}{4 \beta} \bar{g}_{\rho \sigma}\right] \delta R=0 .
\end{aligned}
$$

Imposing $\beta=0$ [after multiplying (2.13) by $\beta$ ], it reduces to the linearized equation around the Schwarzschild black hole found in $f(R)$-gravity [10].

At this stage, we follow the method used in [2] to perform the stability analysis by separating (2.13) into two parts: one is the equation for $\delta \tilde{R}^{\mu \nu}$ as

$$
\left[\bar{g}_{\mu \rho} \bar{g}_{\nu \sigma} \bar{\nabla}^{2}+2 \bar{R}_{\mu \rho \nu \sigma}-\frac{1}{\beta} \bar{g}_{\mu \rho} \bar{g}_{\nu \sigma}\right] \delta \tilde{R}^{\mu \nu}=0 .
$$


The other may be given by taking the trace of (2.13), which leads to (2.9). Hence, we do not consider (2.9) further because it is stable against the perturbation provided $m_{0}^{2}>0$. Actually, this separation was proposed to decouple the massive spin-2 equation from the massive spin-0 equation even though its validity is not yet proved. We may set $\delta R$ to be zero in favor of obtaining equation (2.14) for $\delta \tilde{R}^{\mu \nu}$ which is the key ingredient for describing the massive spin-2 graviton. However, this is not the case unless $\alpha=-\beta / 3$. Hence, for $\alpha=-\beta / 3(\delta R=0)$ [8], Eq.(2.8) could reduce to Eq.(2.14) which becomes a correct linearized equation for $\delta \bar{R}_{\mu \nu}$. We note that the condition of $\alpha=-\beta / 3$ kills a massive spin-0 graviton with mass $m_{0}^{2}$.

Our action (2.1) reveals ghosts when performing the metric perturbation $h_{\mu \nu}$ around the Minkowski spacetimes $\left(M^{4}\right)$ [5]. Explicitly, for choosing $\alpha=-\beta / 3$, its Lagrangian takes the form of $\mathcal{L}=\sqrt{-g}\left[R-\beta\left(R_{\mu \nu}^{2}-R^{2} / 3\right)\right]$. Although the $\beta$-term of providing massive graviton with mass $m_{2}^{2}=1 / \beta$ improves the ultraviolet divergence, it induces ghost excitation [11, 6]

$$
\frac{1}{p^{2}}-\frac{1}{p^{2}+m_{2}^{2}}
$$

which spoils the unitarity but provides a healthy massless graviton. The price one has to pay for making the theory renormalizable is the loss of unitarity. If one is interested in the massive graviton propagation only, it would be better go to the three dimensional new massive gravity [12] where the massive graviton propagates without ghost because of the sign change in $-R$ [13]. However, this is possible in three dimensions only, but it provides a massless graviton with negative norm state in four dimensions

$$
-\frac{1}{p^{2}}+\frac{1}{p^{2}+m_{2}^{2}}
$$

Even though the ghosts (massless graviton) are unavoidable, we may choose ' $-R$ ' instead of $R$ to have a healthy massive graviton in the metric-perturbation theory of the fourth-order gravity. We insist that from (2.14), the Ricci-tensor perturbation $\delta \tilde{R}^{\mu \nu}$ has the massive graviton propagator shown as

$$
\frac{1}{p^{2}+m_{2}^{2}}
$$

around the Minkowski spacetimes.

It is proposed that the ghost issue of the fourth-order gravity persists in the Schwarzschild background. As was pointed out for the Kaluza-Klein compactifications on $M^{4} \times S^{2}$ [14] 
and $M^{4} \times S^{2} \times S^{2} \times S^{2}$ [15], the stability condition is achieved provided that the on-shell amplitude is free from tachyons and ghosts upon plugging the external sources.

In the case of black hole stability, a conventional method of determining the stability is to solve the second-order linearized equation by choosing even-and odd-parity perturbations under the Regge-Wheeler gauge for a massless graviton, which leads to two Schrödinger equations: Regge-Wheeler equation [16] and Zerilli equation [17]. One may conclude that the Schwarzschild black hole is stable because their potentials are positive definite for the whole region outside the black hole, implying that there is no exponentially growing modes with respect to time [1]. If one wants to perform the stability analysis of the Schwarzschild black hole in the fourth-order gravity, one should encounter difficulty in handling fourthorder derivatives in the linearized equation which includes ghost propagations. Up to now, we do not know how to perform the stability analysis of black hole in the metric-perturbation theory of the fourth-order gravity. This is why we introduce the Ricci-tensor perturbation theory which hides the appearance of fourth-order derivatives.

\section{Re-analysis of the black hole stability in fourth- order gravity}

In Einstein gravity, its linearized equation takes a simple form as

$$
\delta R_{\mu \nu}(h)=0 .
$$

Then, the metric perturbation $h_{\mu \nu}$ is classified depending on the transformation properties under parity, namely odd and even. Using the Regge-Wheeler [16] and Zerilli gauge [17], one obtains two distinct perturbations: odd and even perturbations. For odd parity, $h_{\mu \nu}^{o}$ includes two off-diagonal components $h_{0}$ and $h_{1}$ as

$$
h_{\mu \nu}^{o}=\left(\begin{array}{cccc}
0 & 0 & 0 & h_{0}(r) \\
0 & 0 & 0 & h_{1}(r) \\
0 & 0 & 0 & 0 \\
h_{0}(r) & h_{1}(r) & 0 & 0
\end{array}\right) e^{-i k t} \sin \theta \frac{d p_{l}}{d \theta},
$$


while for even parity, the metric tensor takes the form with four components $H_{0}, H_{1}, H_{2}$, and $K$ as

$$
h_{\mu \nu}^{e}=\left(\begin{array}{cccc}
H_{0}(r) e^{\nu(r)} & H_{1}(r) & 0 & 0 \\
H_{1}(r) & H_{2}(r) e^{-\nu(r)} & 0 & 0 \\
0 & 0 & r^{2} K(r) & 0 \\
0 & 0 & 0 & r^{2} \sin ^{2} \theta K(r)
\end{array}\right) e^{-i k t} p_{l}
$$

where $p_{l}$ is Legendre polynomial with angular momentum number $l$ and $e^{\nu(r)}$ is given by (2.4). For $l=0$ (s-mode), $h_{\mu \nu}^{e}$ survives only because of $p_{0}=1$. This mode is responsible for indicating the instability of the black hole in fourth-order gravity. Plugging $h_{\mu \nu}^{o}$ and $h_{\mu \nu}^{e}$ into the linearized Einstein equation (3.1) leads to Regge-Wheeler and Zerilli equations which describe two physical DOF propagating on the black hole spacetimes. Their nonnegative potentials guarantee the stability of the Schwarzschild black hole in the Einstein gravity [18.

In fourth-order gravity with $\alpha=-\beta / 3$, however, we have to solve (2.14) which is rewritten as

$$
\bar{\nabla}^{2} \delta \tilde{R}_{\mu \nu}+2 \bar{R}_{\rho \mu \sigma \nu} \delta \tilde{R}^{\rho \sigma}=m_{2}^{2} \delta \tilde{R}_{\mu \nu}
$$

At this stage, we stress again that (3.4) is considered as the second-order equation with respect to $\delta \tilde{R}_{\mu \nu}$, but not the fourth-order equation for $h_{\mu \nu}^{o / e}$. In massive gravity theory, there is correspondence between linearized Ricci tensor $\delta R_{\mu \nu}$ and Ricci spinor $\Phi_{A B C D}$ when using the Newman-Penrose formalism [19]. Here the null real tetrad is necessary to specify polarization modes of massive graviton, as the four-dimensional massive gravity requires null complex tetrad to specify six polarization modes [20]. This implies that in fourthorder gravity theory, one may take linearized Ricci tensor $\delta R_{\mu \nu}$ (2.11) with 6 DOF $\left[\delta \tilde{R}_{\mu \nu}\right.$ with $5 \mathrm{DOF}$ ] as physical observables, instead of linearized metric tensor $h_{\mu \nu}$ in Einstein gravity [21. Also, we have the tracelessness (2.12) of $\delta \tilde{R}^{\mu}{ }_{\mu}=0$ and the transversality of $\bar{\nabla}^{\mu} \delta \tilde{R}_{\mu \nu}=0$ from the contracted Bianchi identities.

According to Ref. [2] based on (2.14), the author has concluded that the Schwarzschild black hole is classically stable in fourth-order gravity, even though he mentioned a possibility of a static $s$-mode $(\Omega=i k=0, l=0)$ instability for $m_{2}^{2}=1 / \beta<0.19 M_{\mathrm{S}}^{2}=1 /\left(2.294 M_{\mathrm{S}}\right)^{2}$.

Now we are in a position to revisit this conclusion of stability on the Schwarzschild black hole in fourth-order gravity with $\alpha=-\beta / 3$. For this purpose, we stress to note that (3.4) 
takes the following form when replacing $\delta \tilde{R}_{\mu \nu}$ and $m_{2}^{2}$ by $h_{\mu \nu}^{(-)}$and $m^{\prime 2}$ :

$$
\bar{\nabla}^{2} h_{\mu \nu}^{(-)}+2 \bar{R}_{\rho \mu \sigma \nu} h^{(-) \rho \sigma}=m^{\prime 2} h_{\mu \nu}^{(-)}
$$

together with the transverse-traceless condition of $\nabla^{\mu} h_{\mu \nu}^{(-)}=0$ and $h^{(-) \mu}{ }_{\mu}=0$. Surely, Eq. (3.5) is the linearized equation around the Schwarzschild black hole in massive gravity and bimetric theories [3]. The Gregory-Laflamme $(l=0$ mode) instability was recently used to point out the instability of the Schwarzschild black hole in massive gravity and bimetric theories. Considering the perturbations [22]

$$
\left(\begin{array}{ll}
h_{\mu \nu}^{(4)} & h_{\mu z} \\
h_{z \nu} & h_{z z}
\end{array}\right)
$$

around the five-dimensional black string

$$
d s_{5 \mathrm{bs}}^{2}=d s_{\mathrm{S}}^{2}+d z^{2}
$$

$h_{\mu \nu}^{(4)}$ satisfies the massive spin-2 equation (3.5) with $m^{\prime 2}=\mu^{2}$ through $e^{i \mu z}$. It is pointed out that unstable modes of $h_{\mu \nu}^{e}(\underline{3.3}) \rightarrow h_{\mu \nu}^{(-)}(\underline{3.5})$ with $l=0$ and $k=i \Omega$ which are regular at the future event horizon were found within range of $0<m^{\prime}<\frac{\mathcal{O}(1)}{2 M_{\mathrm{S}}}[3]$. Furthermore, the authors [4] have very recently shown that the Schwarzschild black holes are generally unstable against spherically symmetric perturbations in massive gravity and bimetric theories.

Similarly, we find that unstable modes of $h_{\mu \nu}^{e}(\underline{3.3}) \rightarrow \delta \tilde{R}_{\mu \nu}^{e}(3.4)$ with $l=0$ and $k=i \Omega$ where are regular at the future event horizon were found within range of $0<m_{2}<\frac{\mathcal{O}(1)}{2 M_{\mathrm{S}}}$.

Consequently, the Schwarzschild black hole is unstable against the perturbations $\delta \tilde{R}_{\mu \nu}$ in fourth-order gravity with $\alpha=-\beta / 3$ because (3.4) becomes (3.5) when replacing $\delta \tilde{R}_{\mu \nu}$ and $m_{2}^{2}$ by $h_{\mu \nu}^{(-)}$and $m^{\prime 2}$. We note that the instability of Schwarzschild black hole is not proved in fourth-order gravity with arbitrary $\alpha$ and $\beta$ because its linearized equation is given by the complicated form (2.13) which is surely a coupled second-order equation for $\delta \tilde{R}_{\mu \nu}$ and $\delta R$.

Finally, we would like to mention that Whitt [2] has proposed initially a static $(k=0)$ $s$-mode $(l=0)$ instability for $m_{2}^{2}=1 / \beta<0.19 M_{\mathrm{S}}^{2}=1 /\left(2.294 M_{\mathrm{S}}\right)^{2}$ because it indicates a bifurcation of $l=0$ solution of the fourth-order gravity theory. This implies that there is another family of $l=0$ solution, which is identical with the Schwarzschild solution (2.3) at a critical mass $0.44 \beta$. His bound of $0<m_{2}<\frac{1}{2.294 M_{\mathrm{S}}}$ is similar to the instability bound 
of $0<m^{\prime}<\frac{\mathcal{O}(1)}{2 M_{\mathrm{S}}}$. However, he has shown that a static $(k=0) s$-mode $(l=0)$ does not become an unstable mode for $0<m_{2}<\frac{1}{2.294 M_{\mathrm{S}}}$ (see Appendix E in [2]). Actually, the static perturbation has nearly nothing to do with the black hole stability.

Importantly, he has insisted that for non-static $(k \neq 0) s$-mode, there is no unstable perturbations. His flaw seems to arise from the fact that as was shown in Appendix D, he treated the $l=0$ case on equal footing with the $l=1$ case. In the case of massive spin-2 theory, the first instability arises from the $l=0$ perturbation. In other words, he did not solve the $l=0$ perturbation equation correctly. Before a seminal work of the black string stability [22], one does not realize that the $l=0$ perturbation equation reveals an unstable mode.

Furthermore, he has used the simple form (3.4) for arbitrary $\alpha$ and $\beta$ without proving its validity. In this case, (2.13) cannot reduce to (3.4). Thus, his choice of (3.4) is not legitimate. The validity of (3.4) is not proved unless $\alpha=-3 \beta$. Our conclusion on the instability of black hole is suitable for $\alpha=-3 \beta$ case, but not for $\alpha \neq-3 \beta$, where a scalar graviton $\delta R$ with mass $m_{0}^{2}$ propagates around the Schwarzschild black hole spacetimes. Frankly, we have no idea on solving the complicated equation (2.13).

\section{Acknowledgments}

This work was supported by the 2013 Inje University Research Grant.

\section{References}

[1] C. V. Vishveshwara, Phys. Rev. D 1, 2870 (1970).

[2] B. Whitt, Phys. Rev. D 32, 379 (1985).

[3] E. Babichev and A. Fabbri, arXiv:1304.5992 [gr-qc].

[4] R. Brito, V. Cardoso and P. Pani, arXiv:1304.6725 [gr-qc].

[5] K. S. Stelle, Phys. Rev. D 16, 953 (1977).

[6] Y. S. Myung, arXiv:1107.3594 [hep-th].

[7] Y. S. Myung, Eur. Phys. J. C 71, 1550 (2011) [arXiv:1012.2153 [gr-qc]]. 
[8] H. Lu and C. N. Pope, Phys. Rev. Lett. 106, 181302 (2011) arXiv:1101.1971 [hep-th]].

[9] I. Gullu and B. Tekin, Phys. Rev. D 80, 064033 (2009) arXiv:0906.0102 [hep-th]].

[10] Y. S. Myung, T. Moon and E. J. Son, Phys. Rev. D 83, 124009 (2011) arXiv:1103.0343 $[\mathrm{gr}-\mathrm{qc}]]$.

[11] N. H. Barth and S. M. Christensen, Phys. Rev. D 28, 1876 (1983).

[12] E. A. Bergshoeff, O. Hohm and P. K. Townsend, Phys. Rev. Lett. 102, 201301 (2009) arXiv:0901.1766 [hep-th]].

[13] M. Nakasone and I. Oda, Prog. Theor. Phys. 121, 1389 (2009) arXiv:0902.3531 [hepth]].

[14] S. Randjbar-Daemi, A. Salam and J. A. Strathdee, Nucl. Phys. B 214, 491 (1983).

[15] Y. S. Myung, B. H. Cho and Y. -J. Park, Phys. Rev. Lett. 56, 2148 (1986).

[16] T. Regge and J. A. Wheeler, Phys. Rev. 108, 1063 (1957).

[17] F. J. Zerilli, Phys. Rev. Lett. 24, 737 (1970).

[18] S. Chandrasekhar, in The Mathematical Theory of Black Holes (Oxford University, New York, 1983).

[19] E. Newman and R. Penrose, J. Math. Phys. 3, 566 (1962).

[20] D. M. Eardley, D. L. Lee and A. P. Lightman, Phys. Rev. D 8, 3308 (1973).

[21] T. Moon and Y. S. Myung, Phys. Rev. D 85, 027501 (2012) [arXiv:1111.2196 [gr-qc]].

[22] R. Gregory and R. Laflamme, Phys. Rev. Lett. 70, 2837 (1993) hep-th/9301052. 